\title{
STEM/EDS Tomography of Cometary Dust
}

Zack Gainsforth $^{1 *}$, Peter Ercius ${ }^{2}$, Karen Bustillo ${ }^{2}$, Anna L. Butterworth ${ }^{1}$, Christine E. Jilly-Rehak ${ }^{1}$, and Andrew J. Westphal ${ }^{1}$

1. Space Sciences Laboratory, University of California, Berkeley, CA, USA.

2. Molecular Foundry, Lawrence Berkeley National Laboratory, Berkeley, CA, USA.

*Corresponding author: zackg@ssl.berkeley.edu

STEM tomography is rapidly improving with the advancement of instrumentation combined with advanced reconstruction alogrithms such as GENFIRE [1]. Reconstruction of spectroscopic datasets is now possible within reason but not without diÿculty. A primary issue when acquiring STEM/EDS tomographic datasets is the low signal/noise frequently encountered, so a high solid-angle EDS detec-tor is vital. To some extent, noise can be mitigated by optimizing the acquisition and reconstruction pipeline.

For this work, we used the FEI TitanX TEM at the Molecular Foundry at Lawrence Berkeley National Laboratory (LBL) with a 0.6 sr Bruker SDD EDS detector that provided high count rates $\left(\approx 10^{3}-10^{4} \mathrm{cps}\right.$ at $\approx 1 \mathrm{nA}$ ) and a Hummingbird Scientific eucentric tomography holder. We studied an $\approx 500 \mathrm{~nm}$ GEMS (Glass with Embedded Metal and Sulfides, [3]) from a comet retrieved from the stratosphere by a NASA ER-2 airplane (sample: IDP L2071 CL17, particle 3). An important question is whether sulfides reside within the glass matrix of GEMS or on the external surface since this tells us the formation sequence of the phases. Previous tomographic work using HAADF found that sulfides reside on the outside [4], a conclusion which we confirm. Previous 2D studies indicated that GEMS were altered by chemical reactions in the solar nebula, namely sulfides formed subsequent to the formation of the metal/silicate and if so should reside on the outside [5].

We created the tomographic reconstruction in Figure 1 from a sequence of two-minute EDS maps taken every $5^{\circ}$ between $-50^{\circ}$ and $70^{\circ}$ at $80 \mathrm{keV}$ using a beam current of $0.16 \mathrm{nA}$. We processed the maps using software we wrote in python and included as part of the OpenNCEM project on Github [6]. It extracts the HAADF and EDS signals from the maps using the Hyperspy multidimensional data analysis toolbox [7] and separates them into individual stacks. The HAADF stack allows for alignment using an external software of the users' choosing. In our case, we used a pyramid subpixel alignment approach [8] via the StackReg plugin in ImageJ [9]. Our software then applied the alignment to each of the EDS signals and generated scripts for reconstructing them on the Vulcan compute cluster at LBL using GENFIRE. After reconstruction was complete, we visualized the results using ChimeraX [10].

Table 1 shows that the quality of the reconstruction diered significantly depending on whether the element was a majority element or a minority element. This does not address accuracy due to changing geometry while tilting, inability to faithfully extract EDS signals from noisy maps, or other complicating factors. The error gives the percent difference between the counts in each element supplied to the reconstruction algorithm $\left(\Sigma_{\text {in }}\right)$ and the counts in each element after reconstruction $\left(\Sigma_{\text {rec }}\right)$, which likely represents the tomographic reconstruction failing to exactly isolate the correct positional coordinates for each signal. The most abundant signals were C, O, Si, S and Fe. With the exception of Si, these 
had errors $<10 \%$. Other signals had errors ranging up to about $20 \%$. The Si signal teaches us an important lesson. Despite a total of $8.7 \cdot 10^{5}$ counts throughout the entire cube, the silicate was difuse and the noise/pixel was high everywhere. Compare that to $\mathrm{S}$ which had fewer total counts compared to $\mathrm{Si}$ $\left(5.8 \cdot 10^{5}\right)$ yet was significantly less noisy. S resided in small dense sulfide grains so the noise/pixel in pixels containing $\mathrm{S}$ was signficantly lower than for $\mathrm{Si}$, while the background signal for both was comparable. This emphasizes an important point: if phases of interest are well localized, tomography will have a much higher chance of success in identifying those phases.

\section{References:}

[1] Pryor, A., et al. Scientific Reports, 7(10409), (2017), 1-12.

[2] http://www.hummingbirdscientific.com

[3] Bradley, J. P., Science, 265(5174), (1994), 925-929.

[4] Matsuno, J., et al., 77th Meeting of the Meteoritical Society, (2014).

[5] Gainsforth, Z., et al., Lunar and Planetary Science Conference, (2017), 1645.

[6] http://www.github.com/ercius/openNCEM

[7] http://www.hyperspy.org and https://doi.org/10.5281/zenodo.592838

[8] P. Thévenaz, IEEE Transactions on Image Processing, 7(1), 1998, pp. 27-41.

[9] Schneider, C. A., et al., Nature methods 9(7), (2012), 671-675.

[10] Goddard T.D., et al., Protein Sci. 27(1), (2018), 14-25.

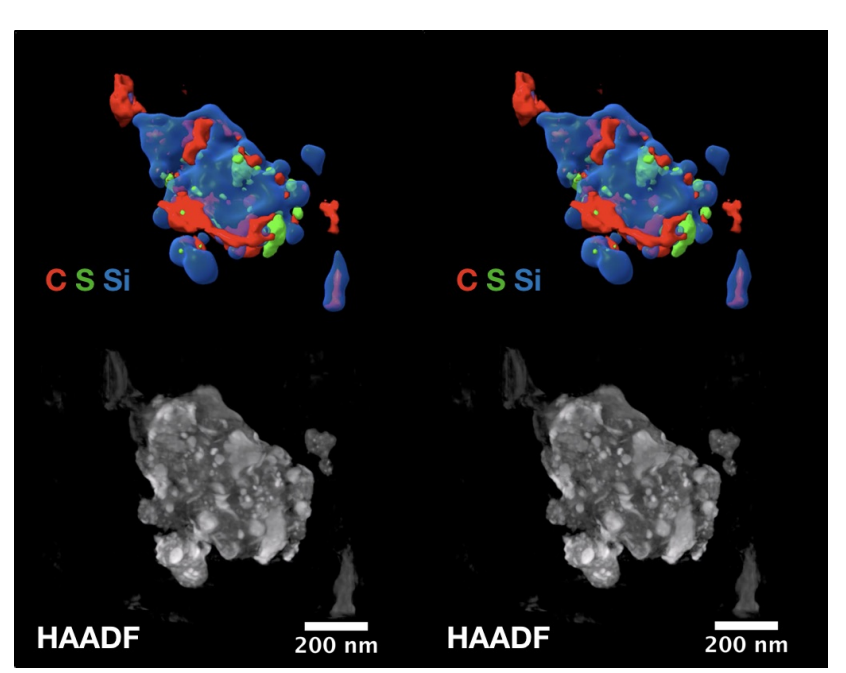

Figure 1. Stereo pairs created in ChimeraX from an EDS tomographic reconstruction of a GEMS from a comet. The top pair shows $\mathrm{S}$ (green), and $\mathrm{C}$ (red) on the surface of the sil-icate material, not inside. A 3 voxel median filter was applied. The bottom pair is a recon-struction from the HAADF signal.

\begin{tabular}{lrrr}
\hline Element & $\Sigma_{\text {in }}$ & $\Sigma_{\text {rec }}$ & $\%$ Err \\
\hline $\mathrm{C}$ & $5 \cdot 10^{6}$ & $5.1 \cdot 10^{6}$ & 3.1 \\
$\mathrm{O}$ & $2.7 \cdot 10^{6}$ & $2.7 \cdot 10^{6}$ & -0.7 \\
$\mathrm{Mg}$ & $2.5 \cdot 10^{5}$ & $3 \cdot 10^{5}$ & 17.2 \\
$\mathrm{Al}$ & $2.2 \cdot 10^{5}$ & $2.6 \cdot 10^{5}$ & 16.4 \\
$\mathrm{Si}$ & $8.7 \cdot 10^{5}$ & $1 \cdot 10^{6}$ & 15.3 \\
$\mathrm{~S}$ & $5.8 \cdot 10^{5}$ & $6.1 \cdot 10^{5}$ & 6.5 \\
$\mathrm{Ca}$ & $3.4 \cdot 10^{5}$ & $3.9 \cdot 10^{5}$ & 13.5 \\
$\mathrm{Fe}$ & $1 \cdot 10^{7}$ & $1 \cdot 10^{7}$ & -4.5 \\
$\mathrm{Ni}$ & $6.8 \cdot 10^{5}$ & $7.2 \cdot 10^{5}$ & 6.3 \\
$\mathrm{Sum}$ & $2.1 \cdot 10^{7}$ & $2.1 \cdot 10^{7}$ & $\mathrm{~N} / \mathrm{A}$ \\
\hline
\end{tabular}

Table 1. Reconstruction statistics behind Figure 1. $\Sigma_{\text {in }}$ gives the total counts input to the reconstruction. $\Sigma_{\text {rec }}$ gives the to-tal counts in the reconstructed EDS cubes (scaled). \% Err shows the difference between the reconstruction and the input. 